\title{
A Strategy for Homogeneous Current Distribution in Direct Methanol Fuel Cells through Spatial Variation of Catalyst Loading
}

\author{
Sang-Min Park ${ }^{1}$, Sang-Kyung Kim ${ }^{2,3, *}$, Dong-Hyun Peck ${ }^{2,3}$, and Doo-Hwan Jung ${ }^{2,3}$ \\ ${ }^{1}$ SFR NSSS System Design Division, Korea Atomic Energy Research Institute, Daedeok-daero 989-111, Yuseong-gu, Daejeon \\ 34057, Republic of Korea \\ ${ }^{2}$ Fuel Cell Laboratory, Korea Institute of Energy Research, 152 Gajeong-ro, Yuseong-gu, Daejeon 34129, Republic of Korea \\ ${ }^{3}$ Major of Advanced Energy and Technology, University of Science \& Technology, 217 Gajeong-ro, Yusong-gu, Daejeon, \\ Republic of Korea
}

\begin{abstract}
A simple strategy is proposed herein for attaining uniform current distribution in direct methanol fuel cells by varying the catalyst loading over the electrode. In order to use the same total catalyst amount for a serpentine flow field, three spatial variation types of catalyst loading were selected: enhancing the cathode catalyst loading (i) near the cathode outlet, (ii) near the cathode inlet, and (iii) near the lateral areas. These variations in catalyst loading are shown to improve the homogeneity of the current distribution, particularly at lower currents and lower air-flow rates. Among these three variations, increased loading near the lateral areas was shown to contribute most to achieving a homogenous current distribution. The mechanism underlying each catalyst loading variation method is different; very high catalyst-loading is shown to decrease the homogeneity of the distribution, which may be caused by water management in the thick catalyst layer thereof.
\end{abstract}

Keywords : Direct methanol fuel cells, Current distribution, Catalyst loading

Received : 22 September 2017, Accepted : 27 November 2017

\section{Introduction}

It has been reported that polymer electrolyte fuel cells (PEFCs), including direct methanol fuel cells (DMFCs), show large deviations in current distribution [1-8]. The deviations are affected by device operating conditions such as the reactant flow rate, reactant concentrations, temperature, and configuration of flow field, as well as by the material properties of the membrane-electrode-assembly (MEA). Deviations from a homogenous current distribution can affect the overall cell performance. Furthermore, continuous deviations can reduce the lifetime of the cell $[5,7]$. Therefore, it is necessary to alleviate the inhomogeneity of current distribution.

*E-mail address: ksk@kier.re.kr

DOI: https://doi.org/10.5229/JECST.2017.8.4.331
In spite of the necessity, few studies have been reported on methods to reduce the inhomogeneity of current distribution. One such study by Santis et al. investigated the homogeneity of current distribution in a hydrogen-fueled polymer electrolyte fuel cell [9]. In this investigation, redistribution of catalyst loading along the channel was achieved to realize homogenous current densities in these devices. A mild or steep increase in catalyst loading along the channel was found to be effective. Studies on the variation in catalyst loading have also been conducted. Wilkinson and St-Pierre [10] reported improved device performance by using an in-plane decreasing gradient of catalyst loading from the cathode inlet to the outlet with three separate sections of different catalyst loadings. However, the homogeneity of the current density was not considered. Prasanna et al. [11] reported improvement in catalyst utilization to reduce the need 
for platinum by using a catalyst-gradient electrode with increased catalyst loading toward the gas outlet. Cell performance was shown to remain unaffected by this loading approach. Prasanna et al. focused on the effects of the gradient method on single cell performance, and did not consider the homogeneity of current density.

In this study, based on our previous results [7], the amount of catalyst loading along the surface of the cathode side was varied to reduce the inhomogeneity of current distributions and to obtain higher cell performance. The strategies used to achieve spatial variation in catalyst loading included increasing the catalyst loading at the cathode side where the current densities were found to be lower in our previous study, whilst maintaining a constant total catalyst loading between the fabricated fuel cells.

\section{Experimental}

\subsection{Current distribution measurement device}

Current Scan Lin of $\mathrm{S}++{ }^{\circledR}$ was used for current mapping. The sensor plate and its segmentations, the locations of the reactant inlet and the product outlet, and the configuration of the five-line serpentine flow field are shown in Fig. 1 and were described in our previous study [7].

The cell was operated in the galvanostatic mode. Unless otherwise specified, the basic operating conditions included a total current of $2 \mathrm{~A}$ or $5 \mathrm{~A}$ loaded onto a single cell, stoichiometric factors $(\lambda)$ set at 5 for the anode and varied between 2 and 10 for the cathode, methanol concentration of $1 \mathrm{M}$, and a cell temperature of $60^{\circ} \mathrm{C}$. The current was loaded onto a single cell for 2 min with a simultaneous measurement of the cell voltage. The current distributions and the cell voltages presented in Section 3 of this work

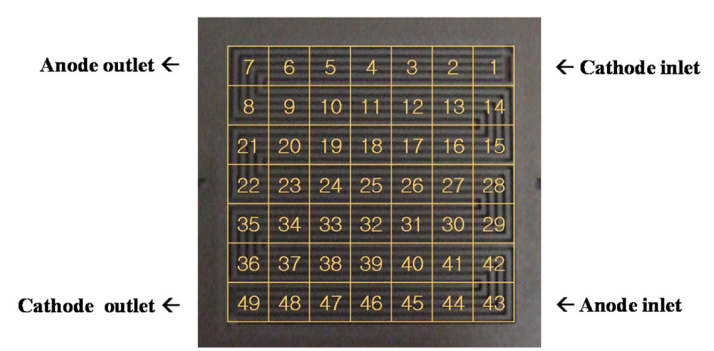

Fig. 1. Segment positions of the measurement cell along the channel [7]. were recorded $100 \mathrm{~s}$ after the current was loaded onto the single cell.

\subsection{Preparation of the MEA}

The catalysts used as the anode and the cathode were PtRu black (HiSpec $6000^{\mathrm{TM}}$, Johnson Matthey) and Pt black (HiSpec $1000^{\mathrm{TM}}$, Johnson Matthey), respectively. To prepare the anode catalyst slurry, 10 $\%$ wt of Nafion ${ }^{\circledR}$ ( $5 \mathrm{wt} \%$ Nafion solution) was added to PtRu black. To prepare the cathode catalyst slurry, $7 \mathrm{wt} \%$ of $\mathrm{Nafion}^{\circledR}\left(5 \mathrm{wt} \% \mathrm{Nafion}^{\circledR}\right.$ solution) was added to Pt black. The anode catalyst slurry was brushed onto the electrode (TGP-H-060, Toray) at a loading of $4 \mathrm{mg} \cdot \mathrm{cm}^{-2}$ of $\mathrm{Pt}$. The different procedures outlining the loading of the cathode catalyst are detailed later. The membrane electrode assembly was prepared by placing a Nafion ${ }^{\circledR} 115$ (Du Pont) membrane between the two electrodes and hot-pressing these three components at $150^{\circ} \mathrm{C}$ for 1 min under a pressure of $70 \mathrm{kgf} \cdot \mathrm{cm}^{-2}$. The final active area of the MEA used was $25 \mathrm{~cm}^{2}(5 \mathrm{~cm} \times 5 \mathrm{~cm})$.

\subsection{Spatial variations in catalyst loading}

Catalyst loadings of different fabricated fuel cells were varied in accordance with the schematics presented in Fig. 2. T4 indicates that a catalyst with 4 $\mathrm{mg} \cdot \mathrm{cm}^{-2}$ of Pt was loaded on to the cathode electrode (SIGRACET ${ }^{\circledR}$ GDL 25BC, SGL Technologies $\mathrm{GmbH})$. U3D5 indicates that the catalyst with $3 \mathrm{mg} \cdot \mathrm{cm}^{-2}$ of Pt was loaded on the upper half of the cathode electrode, and $5 \mathrm{mg} \cdot \mathrm{cm}^{-2}$ of Pt was loaded on the lower half of the cathode electrode. For U2D6, the catalysts with 2 and $6 \mathrm{mg} \cdot \mathrm{cm}^{-2}$ of Pt were loaded (a)

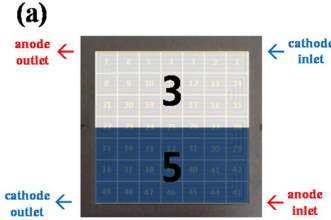

(c)

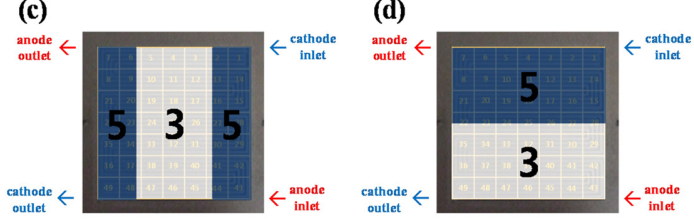

Fig. 2. Schematics of spatial variations in catalyst loading on the cathode side: (a) U3D5, (b) U2D6, (c) L5M3R5, and (d) U5D3. 
on the upper and lower halves of the cathode electrode, respectively. For U5D3, the catalysts with 5 and $3 \mathrm{mg} \cdot \mathrm{cm}^{-2}$ of Pt were loaded on the upper and lower halves of the cathode electrode, respectively. Finally, in case of L5M3R5, the catalyst with $5 \mathrm{mg} \cdot \mathrm{cm}^{-2}$ of Pt was loaded on the left and right quarters of the cathode electrode, and $3 \mathrm{mg} \cdot \mathrm{cm}^{-2}$ of Pt was loaded onto the center half. For all five types of catalyst loading considered, the average loading remained constant at $4 \mathrm{mg} \cdot \mathrm{cm}^{-2}$ of Pt.

\section{Results and Discussion}

The current distributions at different air-flow rates of the cell with a current loading of $2 \mathrm{~A}$ and catalyst loadings as described in Fig. 2 are presented in Fig. 3, 4 , and 5 . The current density for the T4 cell was observed to be lower near the cathode outlet $[3,5,7]$. This observation was found to be more apparent with low air-flow rates at a low applied current of $2 \mathrm{~A}$. This inhomogeneity in current density was alleviated by the catalyst loading of U3D5, and it noticeably reduced for the catalyst loadings for L5M3R5 and U5D3. For U2D6, in contrast, the inhomogeneity of the current density worsened. The "alleviating" and "worsening" mechanisms are different for each catalyst loading and are detailed below.

The current distribution profiles of U3D5 were measured to be different from those measured for the T4 cell. This difference in the current distribution profile can be ascribed to the increase in the catalyst loading near the cathode outlet. In this case, even though water flooding remains important near the cathode outlet, the increased catalytic active area at the lower half with $5 \mathrm{mg} \cdot \mathrm{cm}^{-2}$ of $\mathrm{Pt}$ increases the reaction rate at this region $[10,11]$. For U3D5, therefore, the current densities near the cathode outlet are slightly higher than those for T4, as shown in Fig. 3 (a), (b), and (c). It was also found that the cell voltage enhanced at all cathode flow rates for this catalyst loading (Fig. 3) [7,11]. However, the catalyst loading of U2D6 had an opposing effect on the current distribution. The inhomogeneity of the current distribution with U2D6 was measured to be worse than that measured for T4, resulting in a decrease in the cell voltage (Fig. 3). This inhomogeneity in the current distribution for U2D6 may be ascribed to the thick catalyst layer at the lower half of the cell with a cat-
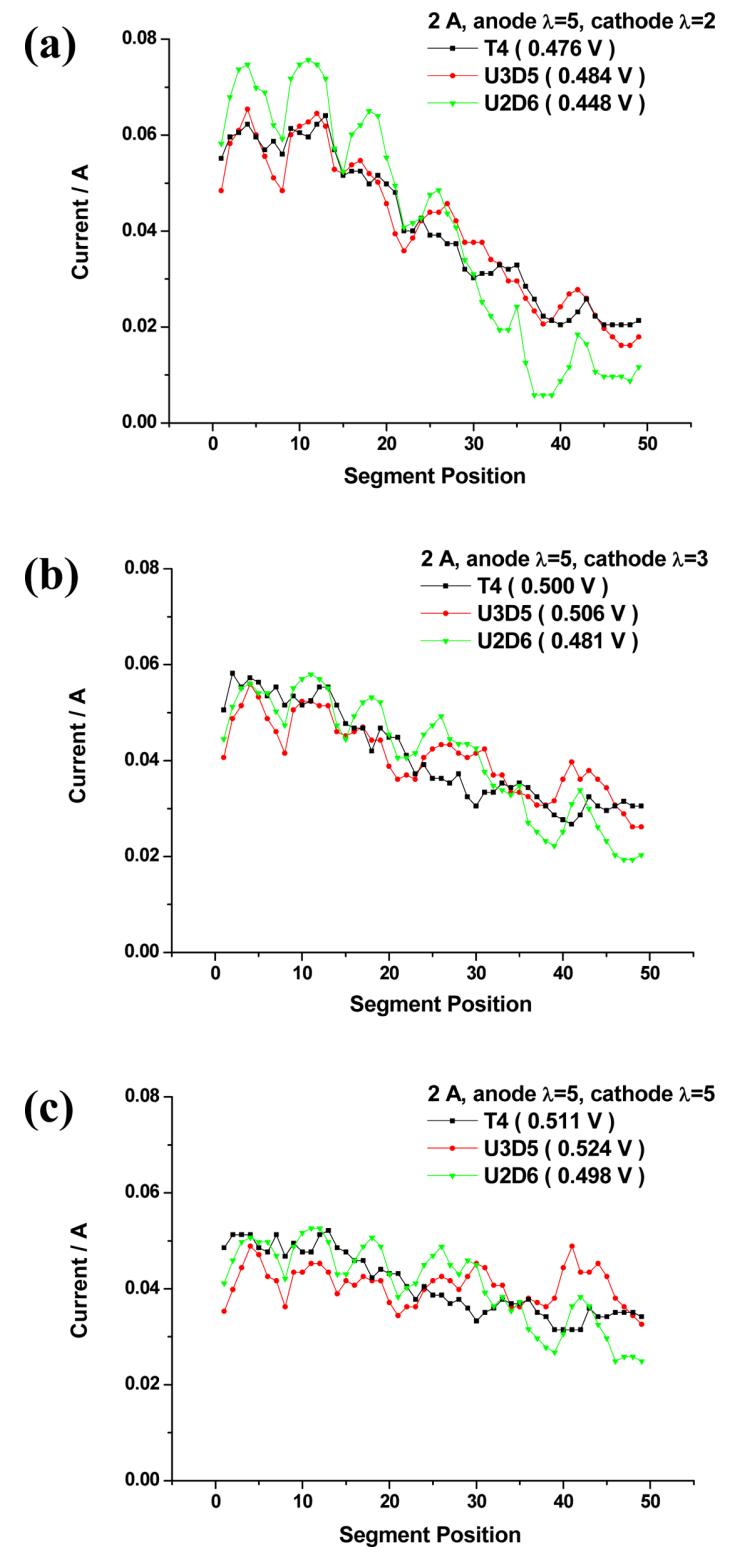

Fig. 3. Current distributions and the corresponding voltages for T4, U3D5, and U2D6 with a loaded current of $2 \mathrm{~A}$ at different cathode flow rates $(\lambda)$ of (a) 2, (b) 3, and (c) 5 .

alyst loading of $6 \mathrm{mg} \mathrm{Pt} \cdot \mathrm{cm}^{-2}$. Thick catalyst layers can increase the mass transport resistance; thus, oxygen transport through the catalyst layer is hindered and local water content cannot be easily removed. As a result, current densities at the thick region reduced. In addition, the periodic drops near 
(a)

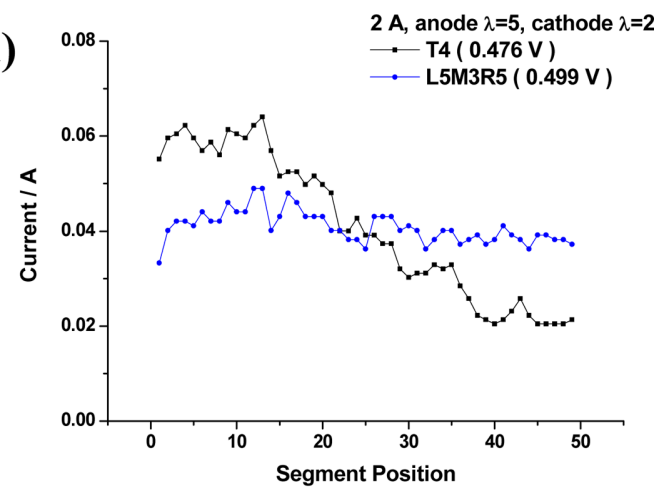

(b)

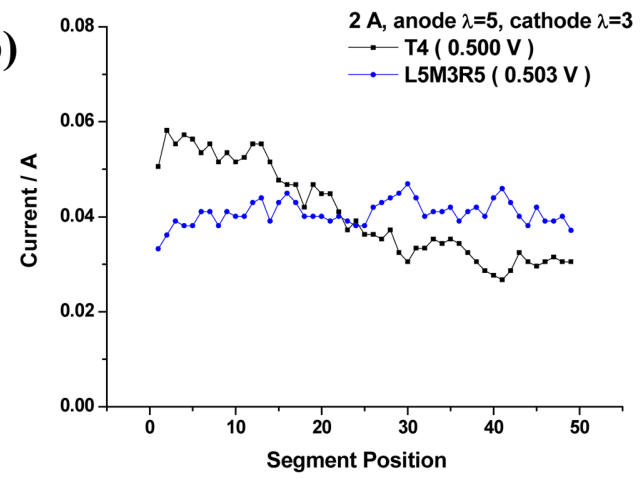

(c)

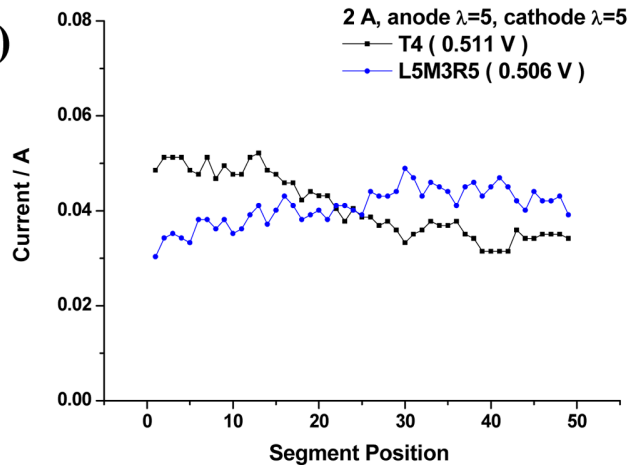

Fig. 4. Current distributions and the corresponding voltages for T4 and L5M3R5 with a loaded current of $2 \mathrm{~A}$ at different cathode flow rates ( $\lambda$ ) of (a) 2, (b) 3, and (c) 5 .

the U-bend of the cathode inlet are more clearly observed, which suggests that water management with this catalyst loading is poor [7].

The homogeneity of the cell current distribution is improved by L5M3R5 loading (Fig. 4). This improvement is more evident at the lower air-flow rates. To increase the active catalyst area in this region, cata- (a)

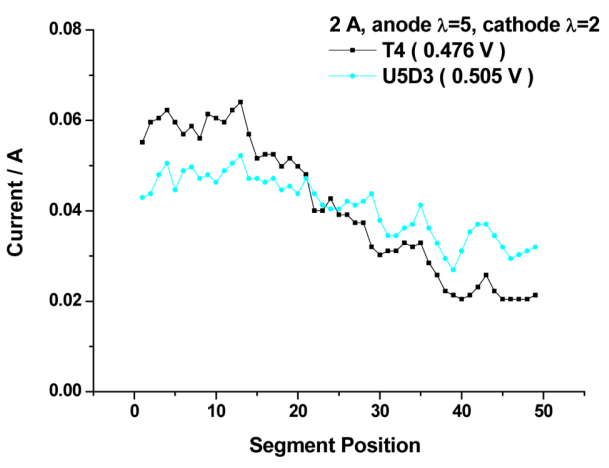

(b)

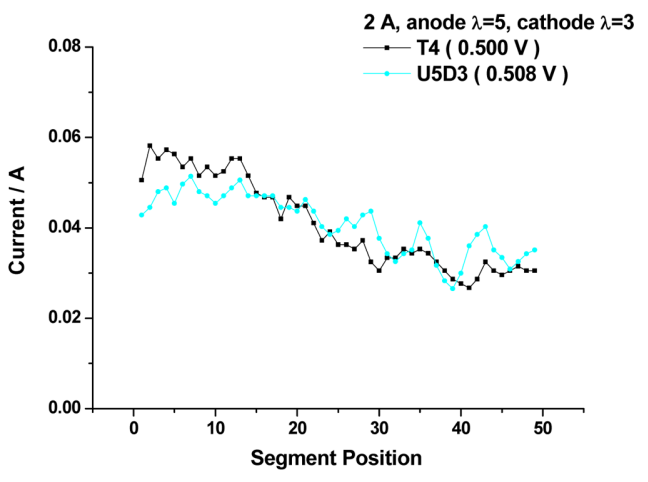

(c)

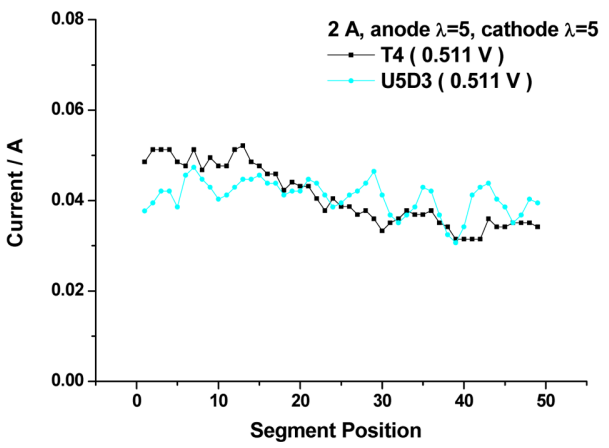

Fig. 5. Current distributions and the corresponding voltages for T4 and U5D3 with a loaded current of $2 \mathrm{~A}$ at different cathode flow rates ( $\lambda$ ) of (a) 2, (b) 3, and (c) 5 .

lyst loading near the U-bend where the current densities periodically drop is enhanced in L5M3R5 [7]. As expected, the periodic drops are not observed with this catalyst loading. Moreover, the current distribution of the L5M3R5 cell has a homogenous current distribution profile, which may be attributed to the periodic enhancement of the catalyst loading. Even though the oxygen mass fraction decreases as the air goes through the channel toward the outlet, the cata- 
lyst loading regularly increased at the region near the U-bend along the air path, thus increasing the catalytic active area at this region. The increase in the catalytic active area of this cell subsequently prevents any decrease in the current density toward the cathode outlet. In addition to the homogenization of current distribution, the cell voltage was also enhanced by the L5M3R5 loading at a low air-flow rate $(\lambda=2)$.

The homogeneity of the current distribution was also shown to be increased by the U5D3 catalyst loading approach (Fig. 5). This approach involves the opposite catalyst loading to that of U3D5. However, the "alleviating" effect on the current distribution profile is more evident for this cell. Wilkinson et al. [10] reported a similar approach with regard to the catalyst loading variation that led to the previously reported improvement in the cell performance of hydrogen-fueled PEFCs. In their work, Wilkinson et al. explained that this approach reduces both kinetic and mass transfer losses near the cathode outlet, where liquid water mass transfer is most prevalent. The catalyst loading is reduced at the cathode outlet so that the oxygen mass transfer rates increase. Similar current distribution mechanisms were observed in this study.

Current distributions at higher catalyst loading with an applied current of $5 \mathrm{~A}$ are presented in Fig. 6 . Compared to those at $2 \mathrm{~A}$, the current distributions were more consistent at $5 \mathrm{~A}$. At the high applied current of $5 \mathrm{~A}$, the $\mathrm{T} 4$ cell is shown to have a homogenous current distribution regardless of the air-flow rate [7]. As a general summary, there did not appear to be any improvement in the homogeneity of the current distribution for all catalyst loadings at the higher loading currents $(5 \mathrm{~A})$. The current densities with the U2D6 catalyst loading remained inhomogeneous in distribution. For L5M3R5, we observed a slight increase in current density toward the cathode outlet (Fig. 6 (b)), which became more obvious as the cathode flow rate increased. Therefore, we can say that the strategy of varying the catalyst loading is more effective in maintaining a homogenous current distribution at lower air flow rates and lower applied currents.

To compare the overall effects of the spatial variations in catalyst loading to one another, the half-sum ratio is defined as the ratio of the half-sum current densities near the cathode outlet to those near the cathode inlet:
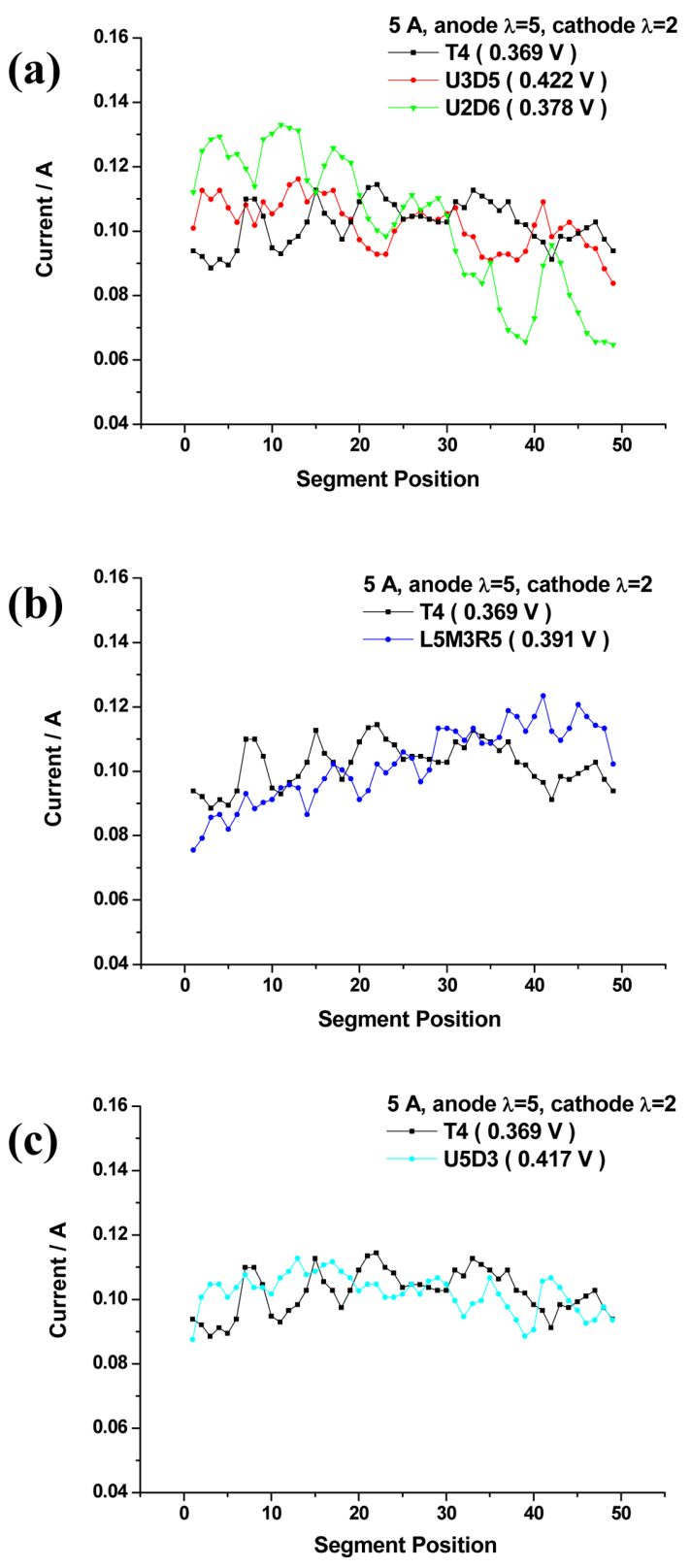

Fig. 6. Comparisons of the current distributions and the corresponding voltages of $\mathrm{T} 4$ with those of (a) U3D5 and U2D6, (b) L5M3R5, and (c) U5D3 with a loaded current of $5 \mathrm{~A}$ at the lowest cathode flow rate $(\lambda)$ of 2 .

$$
\begin{gathered}
\text { half-sum ratio }=\frac{C_{\text {outlet }}}{C_{\text {inlet }}} \\
\text { where } C_{\text {inlet }}=\sum_{n=1}^{n=21} C_{n}+0.5 \times \sum_{n=22}^{n=28} C_{n}
\end{gathered}
$$




$$
C_{\text {outlet }}=\sum_{n=29}^{n=49} C_{n}+0.5 \times \sum_{n=22}^{n=28} C_{n}
$$

$C_{n}$ is the current measured at the $\mathrm{n}^{\text {th }}$ segment position.

The normalized standard deviation is defined as the standard deviation of the current distribution from the average divided by each loaded current ( $2 \mathrm{~A}$ and $5 \mathrm{~A}$ ) [8]. Fig. 7 summarizes the standard deviations of the current density and the half-sum ratios for the five spatial variations of catalyst loading as a function of the cathode flow rate. These values were calculated from the data shown in Fig. 3 to Fig. 6 (current distributions at $2 \mathrm{~A}$ for $\lambda=10$ and those at $5 \mathrm{~A}$ for $\lambda=3,5$, and 10 have not been shown in this article). U3D5, L5M3R5, and U5D3 have lower standard deviations than $\mathrm{T} 4$ at a current load of $2 \mathrm{~A}$ for all the examined cathode flow rates. Among these three loading variations, the deviation for L5M3R5 was lowest at the low air flow rates of $\lambda=2$ and 3 . The standard deviations of T4, U3D5, and U5D3 were similar at the loaded current of $5 \mathrm{~A}$ for all flow rates, as seen in Fig. 7 (b). This result suggests that the variations in the catalyst loading are not effective at the high loaded current of $5 \mathrm{~A}$, which may be attributed to the rela- tively high air flow rate needed to satisfy the required stoichiometric factors. The half-sum ratios at $2 \mathrm{~A}$ were nearest to unity (marked with a dotted line) when L5M3R5 was used at $\lambda=2$ and 3 and when U3D5 and U5D3 were used at $\lambda=5$ and 10. The halfsum ratios at $5 \mathrm{~A}$ were nearest to unity for T4, U3D5, and U5D3 at all cathode flow rates examined. The ratios for L5M3D5 were higher than unity at all flow rates, and those for U2D6 were lower than unity at all flow rates.

\section{Conclusions}

Spatial variation of catalyst loading was performed to reduce inhomogeneous current distributions in direct methanol fuel cells. Our strategy was shown to be successful, and increases in cell voltage were observed for different catalyst loadings. The improvement in the homogeneity of the current distributions is more evident at lower air-flow rates and lower loaded currents. This increased improvement is the result of decreased oxygen concentration at the cathode. Subsequent water flooding is shown to have a greater impact at lower air-flow rates. Most notice- (a)

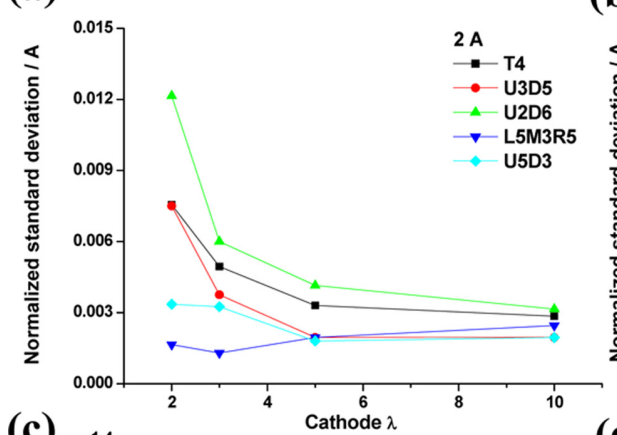

(c)

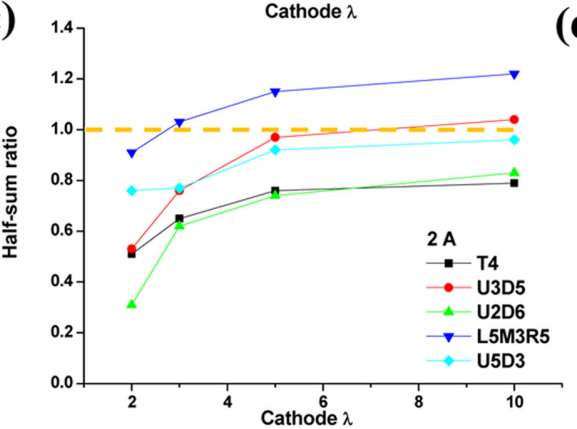

(b)

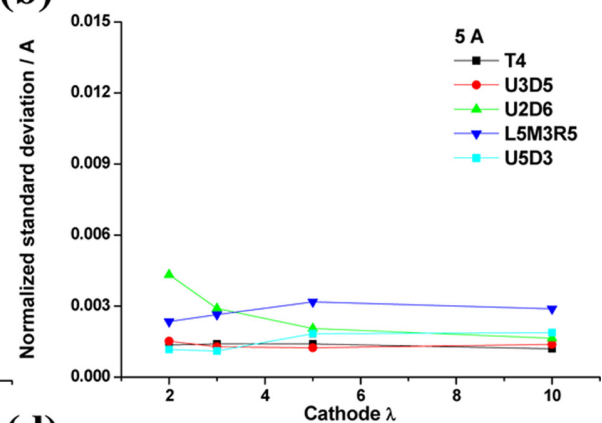

(d)

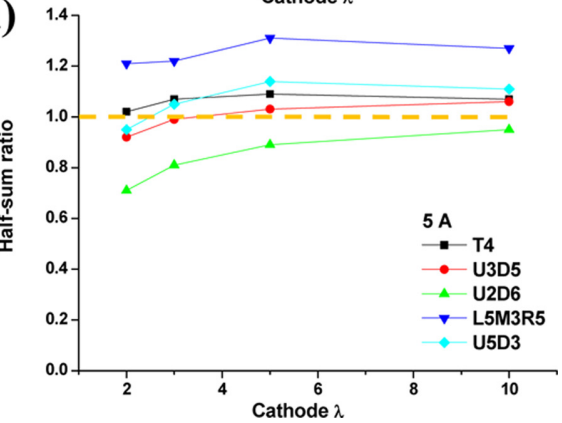

Fig. 7. Standard deviations at (a) $2 \mathrm{~A}$ and (b) $5 \mathrm{~A}$ and half-sum ratios at (c) $2 \mathrm{~A}$ and (d) 5 A for T4, U3D5, U2D6, L5M3R5, and U5D3 as a function of the cathode flow rate from the data of Fig. 3 to Fig. 6. 
able improvement with catalyst loading was observed in the cell referred to as L5M3R5. This cell involved a Pt catalyst loading of $5 \mathrm{mg} \cdot \mathrm{cm}^{-2}$ loaded onto the left and right quarters of the cathode electrode, and $3 \mathrm{mg} \cdot \mathrm{cm}^{-2}$ of the Pt catalyst loaded onto the center half of the electrode. Conflicting observations were noted for durability with spatial variation of catalyst loading on the same electrode. Spatial variation of catalyst loading can enhance the fuel cell durability by making the current distribution consistent. However, non-uniform catalyst loading induces other physical non-uniformity in the electrode such as thickness, porosity, and interface, which can decrease the fuel cell durability. This study reports a simple idea to make the current distribution homogenous in DMFCs, and hence, it provides a procedure to optimize fuel cell applications.

\section{Acknowledgment}

This work was supported by the Korea Institute of Energy Technology Evaluation and Planning (KETEP) and the Ministry of Trade, Industry \& Energy (MOTIE) of the Republic of Korea (No. 20143030031330).

\section{References}

[1] M. Noponen, T. Mennola, M. Mikkola, T. Hottinen, P. Lund, J. Power Sources, 2002, 106(1-2), 304-312.

[2] M. Mench, C. Y. Wang, J. Electrochem. Soc., 2003, 150(1), A79-A85.

[3] H. Sun, G. Zhang, L. J. Guo, H. Liu, J. Power Sources, 2006, 158(1), 326-332.

[4] V. Saarinen, O. Himanen, T. Kallio, G. Sundholm, K. Kontturi, J. Power Sources, 2007, 163(2), 768-776.

[5] F. Ay, A. Ata, H. Dohle, T. Sener, H. Gorgun, J. Power Sources, 2007, 167(2), 391-397.

[6] A. A. Kulikovsky, H. Schmitz, K. Wippermann, Electrochemical and Solid-state Lett., 2007, 10, B126B129.

[7] S.-M. Park, S.-K. Kim, S. Lim, D.-H. Jung, D.-H. Peck, W.-H. Hong, J. Power Sources, 2009, 194(2), 818-823.

[8] S.-M. Park, S.-K. Kim, S. Lim, D.-H. Jung, D.-H. Peck, W.-H. Hong, J. Power Sources, 2011, 196(15), 61106117.

[9] M. Santis, S. A. Freunberger, A. Reiner, F. N. Buchi, Electrochim. Acta, 2006, 51(25), 5383-5393.

[10] D. P. Wilkinson, J. St-Pierre, J. Power Sources, 2003, 113(1), 101-108.

[11] M. Prasanna, E. Cho, H.-J. Kim, I.-H. Oh, T.-H. Lim, S.-A. Hong, J. Power Sources, 2007, 166(1), 53-58. 